\title{
Background Subtraction Using Running Gaussian Average and Frame Difference
}

\author{
Zhen Tang, Zhenjiang Miao, and Yanli Wan \\ Institute of Information Science, \\ Beijing JiaoTong University, Beijing 100044, P.R. China \\ $\{06120376, z j m i a o\} @ b j t u . e d u . c n$, \\ wanlili3646@sohu.com
}

\begin{abstract}
Background Subtraction methods are wildly used to detect moving object from static cameras. It has many applications such as traffic monitoring, human motion capture and recognition, and video surveillance. It is hard to propose a background model which works well under all different situations. Actually, there is no need to propose a pervasive model; it is a good model as long as it works well under a special situation. In this paper, a new method combining Gaussian Average and Frame Difference is proposed. Shadow suppression is not specifically dealt with, because it is considered to be part of the background, and can be subtracted by using an appropriate threshold. At last, a new method is raised to fill small gaps that the detected foreground or the moving objects may contain.
\end{abstract}

\section{Introduction}

Background Subtraction is the first and impotent step in many computer vision applications such as moving object detection, people tracking, traffic monitoring, video surveillance and video semantic annotation. In order to robustly track the moving object, an accurate, reliable and flexible background model is required. As raised in paper [1], a good background model must have the following features: high precision; with the two meanings of accuracy in shape detection and reactivity to changes in time; flexibility in difference lighting conditions; and efficiency in order to provided in real-time. Many background subtraction methods have been proposed in the past decades including Running Gaussian Average , Temporal Median Filter , Mixture of Gaussians, Kernel Density Estimation (KDE), Kalman Filter , and Cooccurence of Image Variations. These methods are either too time consuming (like GMM with online EM algorithm) or too space consuming (like Temporal Median Filter proposed in [3]). Running Gaussian Average is a simple method to describe a background, and it can get a real-time performing. But the shortcoming is it is not a so accurate one. Many methods have been proposed to improve the performing of this method. Koller et al. in [4] remarked that the model should be selectively updated. Sumer Jabri et al. in [5] combined this method with edge information to improve the quality and reliability of the results. In this paper, we combine this method with frame difference method, and 
we propose a new method to fill small gaps that the foreground or the moving objects may contain. In shadows and ghosts suppression aspect, R. Cucchiara et al. in [1] [6] proposed some effective methods, and got good results. In this paper, we consider shadows as part of the background based on the assumption that the shadow is not strong, so we can eliminate it using an appropriate threshold during the background updating period.

The rest of the paper is organized as follows. In section 2, Running Gaussian Average method is summarized. Frame difference method is discussed in detail in section 3 . Then the experiment results and conclusion are discussed.

\section{Running Gaussian Average Model and Frame Difference}

As described by Wren et al. in [2] [5], the background is modeled based on ideally fitting a Gaussian probability density (pdf) on the last $n$ pixel's value. For each pixel, a running average $m_{t}$ and a standard deviation $\sigma_{t}$ are maintained for each color channel. A difference image $D_{t}$ is derived by subtracting the running average from a new coming frame. After a confidence normalization step for every color channel, the image is changed into gray scale and then a binary mask $B^{c}{ }_{t}$ is derived.

The Frame Difference Method [7] is based on the fact that these is nearly no variation of background in consecutive two or three frames. So the moving objects can be simply extracted by the difference of the current frame and the previous frame. In order to eliminate the ghost in the difference images, an intersection step is performed on the consecutive two binarilized difference images. After the above procedure, a binary mask $B_{t}^{f}$ is derived.

\section{The Combination of the Two Methods}

The motivation of combining the two methods is that the masks witch we get above can compensate each other. So we integrate $B_{t}^{f}$ with $B_{t}^{c}$ and get a more reliable one:

$$
B_{t}=\left\{\begin{array}{cc}
0 & B^{c}{ }_{t}+B^{f}{ }_{t}<0 \\
B^{c}{ }_{t}+B^{f}{ }_{t} & 0 \leq B^{c}{ }_{t}+B^{f}{ }_{t} \leq 255 \\
255 & B^{c}{ }_{t}+B^{f}{ }_{t}>255
\end{array}\right.
$$

Sometimes $B_{t}$ has some small gaps or even big holes after filtering the noises, so we employ the following steps to fill the gaps and holes. 
step1: filling small gaps

A $3 \times 3$ or $5 \times 5$ window is employed to remove the small gaps [7]. We set each foreground pixel at the center of the window, and if there are more than half of the foreground pixels contained in the window, then we will fill the gaps within the window. This method is effective to the small gaps, but it is helpless with the big holes. Then step2 is performed.

step2: filling big holes

Actually, holes are part of foreground regions, but misplaced as the background. In order to check if a background pixel is misplaced or not, we employed a larger $25 \times 25$ window. Set each background pixel at the center of the window, and check if there are enough foreground pixels at each direction of eight directions (north, south, east, west, northeast, northwest, southeast and southwest), if there are, then this pixel belongs to the foreground, and we give it the value 255 .

\section{Experiment Results and Conclusions}

In our experiment, the proposed method is compared with the running Gaussian average and the frame difference, and the results are shown in Fig.1.

From the experimental results, we can see that, the result of the running Gaussian average $B^{c}{ }_{t}$ is no so satisfied, many pixels of the moving object is wrongly detected; the edge of the object is not consecutive too. The frame difference has a better result $B^{f}{ }_{t}$, but is also has some gaps and holes, which should be removed from the masks. The result of the proposed method is shown in Fig.1. (c), which is more accurate and reliable. Fig.1. (d) is the result after filling the gaps and holes.

But when a moving object stops, it will be quickly incorporated into the background. This may result in object losing when used to object tracking application. And how to further eliminate the noise is also a challenging problem.

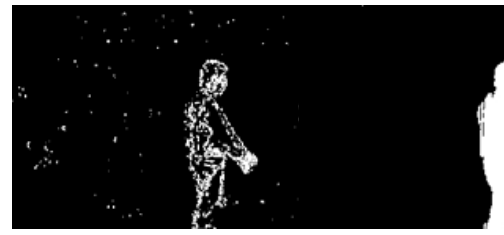

$\begin{array}{ll}\text { (a) } B_{t}^{c} & \text { (b) } B_{t}^{f}\end{array}$

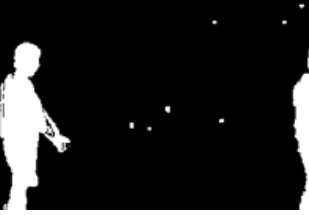

(c) $B_{t}$

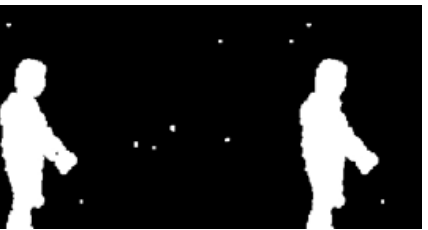

(d) after filling the holes

Fig. 1. Experiment results 


\section{Acknowledgments}

This work is supported by National 973 Key Research Program 2006CB303105, National 973 Key Research Program 2004CB318110 and University Key Research Fund 2004SZ002.

\section{References}

1. Cucchiara, R., Piccardi, M., Prati, A.: Detecting Moving Objects, Ghost, and Shadows in Video Streams. IEEE Transactions on Analysis and Machine Intelligence 25(10), 1337-1342 (2003)

2. Wren, C., Azarhayejani, A., Darrell, T., Pentland, A.P.: Pfinder: real-time tracking of the human body. IEEE Trans. on Pattern Anal. And Machine Intell. 19(7), 780-785 (1997)

3. Lo, B.P.L., Velastin, S.A.: Automatic congestion detection system for underground platforms. In: Proc. ISIMP2001, pp. 158-161 (May 2001)

4. Koller, D., Weber, J., Huang, T., Ma, J., Ogasawara, G., Rao, B., Russell, S.: Towards Robust Automatic Traffic Scene Analysis in Real-time. In: Proc. ICPR'94, pp. 126-131 (November 1994)

5. Jabri, S., Durie, Z., Wechsler, H., Rosenfield, A.: Detection and Location of People in Video Images Using Adaptive Fusion of Color and Edge Information. In: icpr, 15th International Conference on Pattern Recognition (ICPR'00), vol. 4, p. 4627 (2000)

6. Cucchiara, R., Grana, C., Piccardi, M., Prati, A.: Detection Objects, Shadows and Ghosts in Video Streams by Exploiting Color and Motion Information. In: 11th International Conference on Image Analysis and Processing, pp. 360-365 (September 26-28, 2001)

7. Zang, Q., Klette, R.: Robust Background Subtraction and Maintenance. In: Proceedings of the 17th International Conference on Pattern Recognition, Cambridge, UK, pp. 90-93 (2004) 